\title{
Níveis de ansiedade estado dos bailarinos de um grupo de dança da cidade de Juazeiro do Norte-CE
}

\author{
Levels of anxiety state of the dancers of a dance group \\ of the city of Juazeiro do Norte-CE
}

\author{
João Paulo da Silva Maciel ${ }^{1}$ \\ Dioneide Pereira da Silva ${ }^{2}$ \\ Thiago Sobreira de Araújo ${ }^{3}$ \\ Rebeca Sampaio Cabral ${ }^{3}$
}

\section{RESUMO}

Introdução: A ansiedade pode ser descrita como a reação natural que impulsiona o ser humano a alcançar seus objetivos. Esse estado emocional pode tornar-se patológico e repercutir de forma negativa se vivenciado excessivamente e por longos períodos. Objetivo: Verificar os níveis de ansiedade estado dos bailarinos da cidade de Juazeiro do Norte-Ceará. Métodos: A pesquisa caracteriza-se por ser um levantamento de estudo quantitativo, descritivo e exploratório, de campo com delineamento de corte transversal. A amostra foi constituída por 26 bailarinos de ambos os sexos, sendo que foram pesquisados $(\mathrm{n}=08)$ sujeitos do sexo masculino referente a $(30,8 \%)$ e $(n=18)$ sujeitos do sexo feminino com percentual de $(69,2 \%)$ com média de idade de $(19,2 \pm 2,26)$. Para a realização da coleta de dados, optou-se pelo inventário de ansiedade estado IDATE. Resultados: Diante os resultados obtidos nesta pesquisa, pode-se perceber que dos vinte cincos indivíduos entrevistados (50,0\%) dos homens e $(16,7 \%)$ das mulheres, se encontrava com baixo nível de ansiedade. Outros pontos observados foram os indivíduos com nível médio de ansiedade que foi $(50,0 \%)$ do sexo masculino e $(66,7 \%)$ do sexo feminino, já na escala de alto nível de ansiedade foi observado que somente $(16,6 \%)$ do sexo feminino apresentaram níveis de ansiedade estado. Conclusões: Conclui-se que acerca dos níveis de ansiedade estado dos bailarinos de um grupo de dança da cidade de Juazeiro do Norte-Ceará, percebe-se que os mesmo apresentam alteração nos estados emocionais antes da apresentação.

\section{PALAVRAS-CHAVE}

Níveis de ansiedade; Ansiedade Estado; Bailarinos; Grupo de dança.

\footnotetext{
${ }^{1}$ Centro Integrado de Tecnologia e Pesquisa (CINTEP). Faculdade Nossa Senhora de Lourdes, Porto Seguro, Bahia

${ }^{2}$ Universidade de Lisboa (Faculdade de Motricidade Humana-FMH)Cruz Quebrada, Portugal.

${ }^{3}$ Centro Universitário Dr. Leão Sampaio (UNILEÃO) Juazeiro do Norte, Ceará, Brasil.
} 


\section{ABSTRACT}

Introduction: Anxiety can be described as the natural reaction that drives humans to achieve their goals. This emotional state can become pathological and have a negative impact if experienced excessively and for long periods. Objective: verify the levels of state anxiety of the dancers of the city of Juazeiro do Norte-Ceará. Methods: the characterized by being a quantitative, descriptive and exploratory field study with a cross-sectional design. The sample consisted of 26 dancers of both sexes, and males $(n=08)$ were surveyed for $(30.8 \%)$ and $(n=18)$ female subjects with a percentage of $(69.2 \%)$ with a mean age of $(19.2 \pm 2.26)$. In order to collect data from this study, we chose the State IDATE anxiety inventory. Results: Given the results obtained in this research, it can be seen that of the twenty-five individuals interviewed (50.0\%) of men and (16.7\%) women, was low in anxiety. Other points observed were individuals with medium anxiety level that was (50.0\%) males and (66.7\%) females, while on the high anxiety scale, it was observed that only (16.6\%) females had levels of state anxiety. Conclusions: It is concluded that about the levels of anxiety state of the dancers of a dance group in the city of Juazeiro do Norte-Ceará, it is perceived that they present alterations in emotional states before the presentation.

\section{KEYWORDS}

Anxiety Levels; State Anxiety; Dancers; Dance group. 


\section{INTRODUÇÃO}

A vida se manifesta através da saúde que são formas únicas, experiências subjetivas e que não podem ser manifestadas integralmente através de palavras. Diante este contexto a Organização Mundial de Saúde (OMS) conceitua a saúde como sendo um estado de completo bem-estar físico, mental e social, e não consiste apenas na ausência de doença ou de enfermidades (BACKES, et. al., 2017).

A saúde como um todo não é apenas a ausência de doenças, pois envolve hábitos relacionados ao comportamento, ao estilo de vida e ao modo de encarar os desafios, ou seja, aspectos relacionados aos fatores emocionais que ocorrem no dia-a-dia. Múltiplos são os fatores que determinam a compreensão da saúde ou enfermidade, comoosfatoresgenéticos,bioquímicos,comportamentais, psicodinâmicos e socioambientais. Esses fatores podem interagir de modo complexo esses que na maioria dos casos podem está relacionados aos níveis de ansiedade pré e pós-apresentações (MELO, 2016).

A ansiedade pode ser descrita como reação natural que impulsiona o ser humano a alcançar seus objetivos, podendo ser apontada por muitos estudiosos como o "mal do século" junto com a depressão, sendo estimada em dez milhões o número de brasileiros afetados por algum tipo de transtorno de ansiedade (CARVALHO; FARAH; GALDEANO, 2017).

A ansiedade é uma característica biológica que antecede situações de risco, seja ele real ou imaginário. Portanto os transtornos de ansiedade prejudicam o trabalho, chegam a impedir as relações interpessoais, atrapalham a vida afetiva. Autores como Spielberg et al., (1970) descreveram o estado de ansiedade como sendo um estado momentâneo, transitório, caracterizado por tensão, apreensão e por elevação das atividades do sistema nervoso autônomo, dependendo da percepção da situação, sendo mais alto o nível de estado de ansiedade quando a situação é percebida como ameaçadora.

Neste contexto a dança pode ser uma ferramenta importante para a manutenção de uma vida saudável, pois proporciona um bom condicionamento físico, auxiliando no desenvolvimento das capacidades físicas, como também proporciona benefícios a nível cardiovascular, melhorando a flexibilidade e coordenação, como também os benefícios psicológicos, que pode incentivar inclusive os indivíduos mais sedentários e diminuir os níveis de ansiedade (BAPTISTA, 2018).

Sabe-se que a dança por ser uma atividade física para todas as pessoas, não existe nenhuma restrição de idade e nem impedimento, já que a sua prática pode ser adaptados às limitações físicas de cada um, na maioria dos casos sua busca e utilizada como meio de diversão e descontração, proporcionando também um ótimo condicionamento físico, dentre outros benefícios (BABTISTA, 2018). Essas mudanças ajudam significativamente no comportamento, ou seja, desenvolvendo o equilíbrio emocional, como tambem a saúde mental e física, mantendo o peso desejado, pelo qual vem sendo um fator decisivo para conquistar um corpo mais saudável (JUSSARA, 2017).

Portanto por estes motivos apresentado que o referido estudo mostra sua relevância em torno do tema abordado, sabendo que cada vez mais as pessoas estão em busca da melhoria da qualidade de vida, e em sua maioria, a encontram a partir dos benefícios proporcionados pela atividade física através da dança, um desses benefícios é a diminuição da ansiedade.

Diante o exposto o referido estudo teve como objetivo geral: Verificar os níveis de ansiedade estado dos bailarinos da cidade de Juazeiro do Norte-Ceará. Buscando neste aspecto identificar a dança como fator de diminuição da ansiedade estado e verificar a presença de alterações nos níveis de ansiedade estado no período pré-palco ou diante da pré-apresentação dos bailarinos.

\section{MATERIAIS E MÉTODOS}

Essa pesquisa caracteriza-se por ser um levantamento de estudo quantitativo, descritivo e exploratório, de campo com delineamento de corte transversal.

“A pesquisa quantitativa em seu contexto considera o que pode ser quantificável, o que significa traduzir em números opiniões e informações para classificá-las e analisá-las (MARCONI; LAKATOS, 2015)”. A integração da pesquisa quantitativa pode aplicar questionários, investigar diferentes questões em diferentes ocasiões, pode utilizar fontes documentais e dados estatísticos. É apropriada para medir tanto opiniões, atitudes e preferências como comportamentos (THOMAS; NELSON, 2016). 
Sendo assim o estudo foi desenvolvido na cidade de Juazeiro do Norte, Ceará, localizado na região do Cariri, cuja população de estudo foi constituída por bailarinos profissionais de uma companhia de dança.

A amostra foi composta por 26 bailarinos de ambos os sexos, sendo pesquisados no total $(\mathrm{n}=08)$ sujeitos do sexo masculino referente à $(30,8 \%)$ e $(n=18)$ sujeitos do sexo feminino com percentual de $(69,2 \%)$ com media de idade de $(19,2 \pm 2,26)$, e que correspondessem ao critério de inclusão: estar com mais de 03 anos de experiência profissional. 0 única critério de exclusão adotado foi estar a menos de 03 anos na companhia de dança.

Os participantes da pesquisa foram selecionados a partir da amostragem por conveniência, considerando que a seleção da unidade amostral a ser pesquisada é mais facilmente acessível e intencional por que os indivíduos pesquisados são selecionados de modo a ser mais próximo do valor médio das variáveis da população (THOMAS; NELSON, 2016).

Para a realização da coleta de dados deste estudo, optou-se pelo inventário de ansiedade estado IDATE, elaborado por Spielberger (1970). Sendo este composto de 20 questões de múltipla escolha para cada tipo de ansiedade (estado), sendo um instrumente de autorrelato com escore para item individual que tem as opções de respostas: 04 - Muitíssimo; 03 - Bastante; 02 - Um pouco; 01- Absolutamente não. 0 questionário IDATE desde 1990 foi reconhecido no Brasil e o mesmo se apresenta em forma de questionário.

Os procedimentos metodológicos da presente pesquisa constituíram-se dentro dos padrões éticos legais referentes à pesquisa com seres humanos da resolução $446 / 12$. A proposta de estudo foi submetida à aprecia- ção ao comitê de ética em pesquisa do Centro Universitario Dr. Leão Sampaio e aprovado sob o parecer de numero 1.035.476.

A aceitação na participação foi permitida mediante a assinatura de um Termo de Consentimento Livre e Esclarecida (TCLE), contendo explicações sobre os objetivos da pesquisa, o caráter voluntário da participação no estudo e do direito de retirar-se da pesquisa quando achar conveniente. Levando em consideração aos procedimentos, o questionário foi aplicado antes do inicio da apresentação do grupo de dança de forma individual para avaliar os níveis de ansiedade "Estado" dos participantes da pesquisa.

Após o recolhimento de todos os questionários foi constituído um banco de dados no programa Excel 2010, para que assim fosse realizada uma analise por meio do software Statistical Package for the Social Sciences (SPSS), versão 20.0, para analise da estatística descritiva e exploração completa dos dados (mínimo, máximo, média, desvio-padrão e o percentual). Para avaliação da medida de significância estatística utilizou-se o teste " $t$ " de Student e o teste de correlação de Pearson Quí-quadrado (para valores esperados menores que 05$)$, adotando $(p<0,05)$, sendo transportado logo após para programa Microsoft Office Word 2010.

\section{RESULTADOS E DISCUSSÃO}

Os dados referentes aos resultados desta pesquisa foram coletados no primeiro semestre de 2019, tabulados separadamente e, posteriormente, criadas as tabelas e figuras para uma melhor interpretação a fim de demonstrar estatisticamente valores referentes à análise dos dados.

Tabela 1. Análise descritiva das perguntas de caráter positivo e negativo do sentimento de ansiedade traço-estado de acordo com a soma das pontuações adquiridas para o sexo masculino e feminino.

\begin{tabular}{cccc}
\hline Sentimento & Medidas de Dispersão & Masculino & Feminino \\
\hline \multirow{3}{*}{$\begin{array}{c}\text { Positivo de ansiedade } \\
\text { estado }\end{array}$} & Mínimo & 14 & 13 \\
& Máximo & 29 & 34 \\
& Média & 21,4 & 25,7 \\
& Desvio padrão & 5,4 & 5,4 \\
Negativo de ansiedade & Mínimo & 17,0 & 15,0 \\
estado & Máximo & 32,0 & 33,0 \\
& Média & 23,5 & 24,1 \\
& Desvio padrão & 5,8 & 4,2 \\
\hline
\end{tabular}

Fonte: Pesquisa 2019 
De acordo com a Tabela 01: A média envolvendo os níveis de ansiedade estado na escala positiva foi $(21,4 \pm$ $5,4)$ para o sexo masculino, seguido de $(25,7 \pm 5,4)$ do sexo feminino. Já referente à escala negativa dos bailarinos do sexo masculino pode-se perceber que a media foi de $(23,5 \pm 5,8)$ e das bailarinas do sexo feminino foi $(24,1 \pm 4,2)$.

Analisando os dados percebe-se que com relação às medias obtidas na escala de sentimentos positivos, os os bailarinos apresentaram-se melhor no sexo feminino, quando comparados com os bailarinos do sexo masculinos. Já na escala de sentimentos negativos foi obser- vado os mesmo resultados, em relação aos achados dos níveis de ansiedade da escala positiva.

Esses dados corroboram com estudos em que autores ao investigarem o nível de estresse e ansiedade estados em bailarinos e bailarinas profissionais na pré-estréia de um espetáculo de dança, encontraram sintomas bem definidos e parecidos com a presente pesquisa em que segundo a pesquisa apontaram também dados referentes à escola de sentimentos positivos e negativos com maior resultado para o sexo feminino, ou seja, significando que a presença de ansiedade estado nas bailarinas foi maior que nos bailarinos antes da apresentação (SOUSA; MARIANI; SAMULSKI, 2016).

\section{Masculino $\quad$ Feminino}

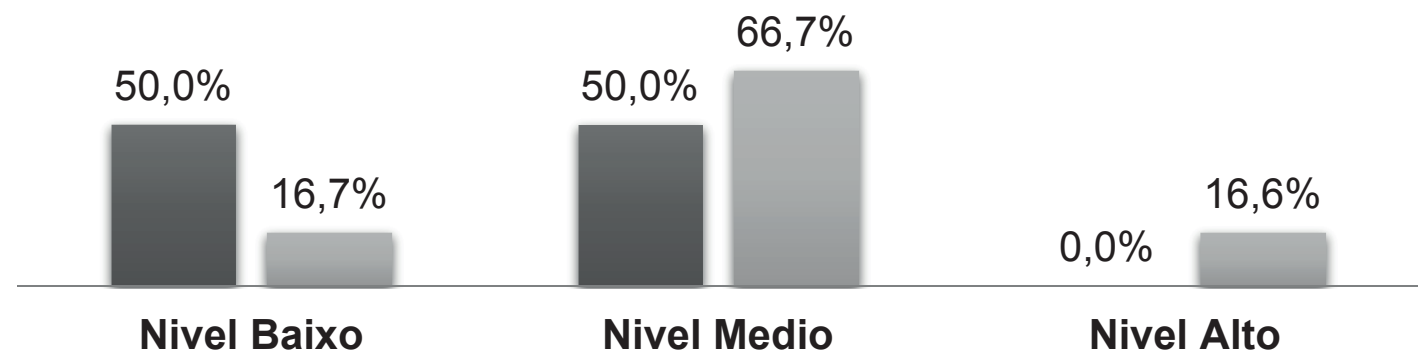

Figura 1. Classificação dos bailarinos de acordo com os níveis de ansiedade estado, pré - apresentação com relação às perguntas de caráter positivo.

Fonte: Pesquisa 2019.

De acordo com a figura 1 que descreve a classificação dos bailarinos de acordo com os níveis de ansiedade estado - pré - apresentação às perguntas de caráter positivo da escala de sentimentos IDATE.

Por essa figura percebe-se que dos vinte e cinco indivíduos entrevistados (50,0\%) de homens estavam com baixo nível de ansiedade e $(16,7 \%)$ mulheres com baixo nível de ansiedade. Outros pontos observados foram os indivíduos com nível médio de ansiedade que foi $(50,0 \%)$ do sexo masculino nessa categoria seguido de $(66,7 \%)$ do sexo feminino, na categoria de alto nível de ansiedade encontra-se apenas (16,6\%) do sexo feminino.

Pode-se analisar que em eventos de dança normalmente os bailarinos apresentam níveis de ansiedade mesmo de caráter positivo, podendo interferir na concentração dos bailarinos e consequentemente gerar estresse de tensão e ansiedade, fator este que pode ser prejudicial para o desempenho de alguns dos bailarinos, assim como para a qualidade de apresentação durante o espetáculo, pois assim pode prejudicar a concentração para desenvolver as coreografias e lembrar o tempo e a batida musical.

Percebe-se então que a ansiedade é um estado emocional que apresenta componentes psicológicos e fisiológicos, fazendo parte da experiência humana. Sendo responsável pela adaptação do organismo a situações de perigo, constituindo uma condição que pode ocorrer a qualquer sujeito (PRIMO; PRADO; PRADO, 2018).

Nos estudos de Sousa, Mariani e Samulski (2016), concluíram que tanto os bailarinos quanto as bailarinas apresentaram sintomas bem definidos que indicaram a presença de estresse e ansiedade por ocasião da pré-estreia de dança. Neste caso as bailarinas no respectivo estudo apresentaram mais sintomas que os bailarinos, porém os mesmos obtiveram níveis de ansiedade médios e elevados. 


\section{Masculino Feminino}

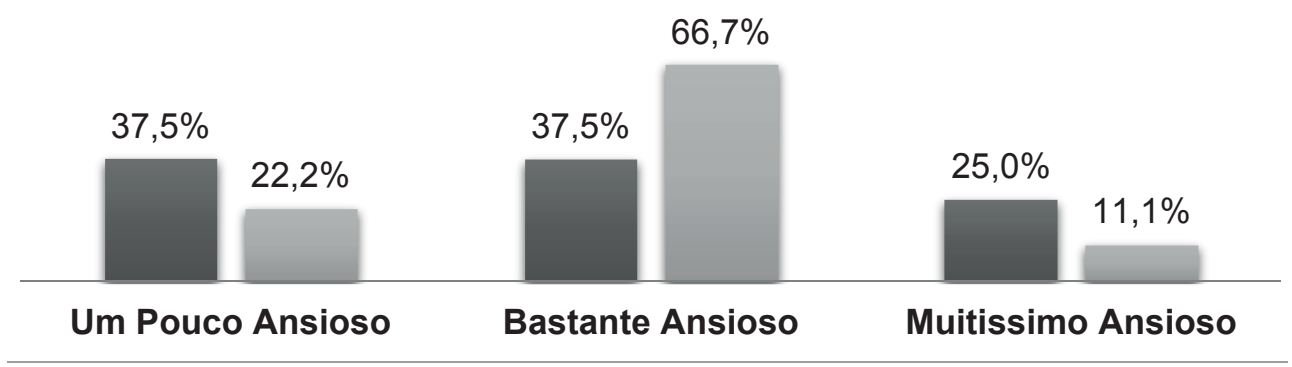

Figura 2. Classificação dos bailarinos de acordo com os níveis de ansiedade estado, pré - apresentação com relação às perguntas de caráter Negativo.

Fonte: Pesquisa 2019

A Figura 02 descreve sobre a classificação dos bailarinos de acordo com os níveis de ansiedade estado, pré - apresentação com relação às perguntas de caráter negativo, percebe-se que dos 25 indivíduos que foram entrevistados $(37,5 \%)$ do sexo masculino apresentava-se "Um pouco ansioso" e $(22,2 \%)$ de sexo feminino estavam nessa categoria.

Já na categoria de "Bastante ansioso" encontrava-se $(37,7 \%)$ do sexo masculino e $(66,7 \%)$ do sexo feminino, ou seja, o sexo feminino de acordo com esta pesquisa apresentou-se bastante ansiosa mais que o sexo masculino.

Foi observado também como descrito a categoria de "Muitíssimo ansioso" (25,0\%) dos homens e (11,1\%) das mulheres, pode-se observar nessa categoria, que os homens encontravam-se antes da apresentação mais ansioso que as mulheres. Outro ponto observado foi que para todas as categorias as mulheres demonstraram mais confiantes que os homens antes da apresentação.

Levando em conta o inventário feito por Spielberger (1970), a ansiedade correspondente nos bailarinos/as durante a pré-apresentação é a ansiedade estado, em que a situação representativa de perigo é a própria apresentação em palco. Porém, cabe lembrar, que uma pessoa com elevado nível de ansiedade-traço vai responder com um alto nível de ansiedade-estado a um número maior de situações de tensão (MAGILL, 1984).

A ansiedade estado é como uma situação provisória, que envolve sentimentos desagradáveis de tensão e pensamentos apreensivos (STRONEMAN,1998). Segundo outros autores a ansiedade estado seria uma reação episódica ou situacional, ou seja, um estado emocional transitório pelo qual o individuo esta passando antes de uma situação de tensão emocional (BARLOW, 2015; BAPTISTA et.al., 2018)

A tabela 2 corresponde aos resultados referentes aos testes de "correlação de Student e Pearson (Quí-Quadrado)". Considerando o " $p$ valor" para o teste $t$ de Student, pode-se observar que houve significância de variância entre as escalas de níveis de ansiedade estado, o que expressa que ambas as variáveis analisadas não apresentaram diferença significativa com relação ao teste para valores esperados $(\mathrm{p}<0,05)$.

Tabela 2. Distribuição do Teste de $t$ de Student, relações estatisticamente significantes entre as escalas de caráter positivo e negativo para o sexo masculino e feminino.

Masculino Feminino

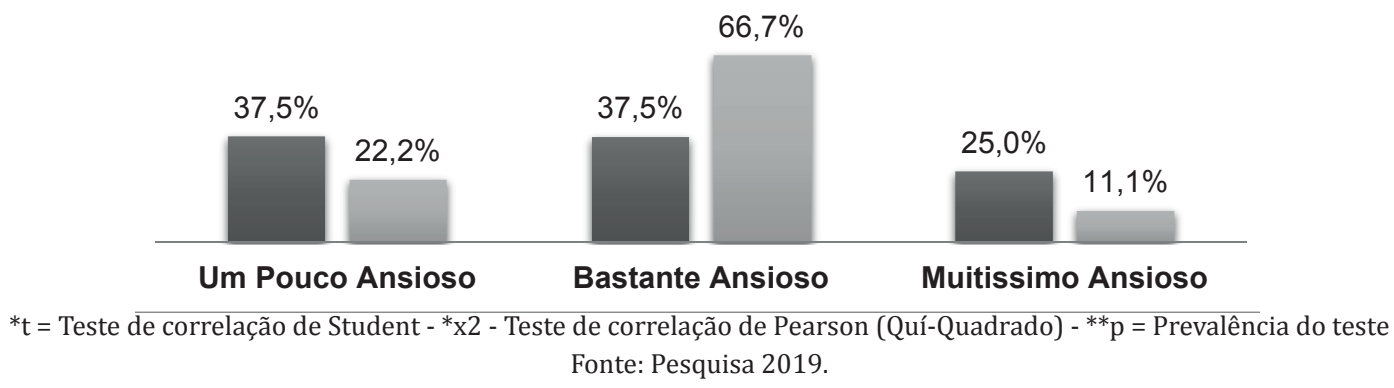

Fonte: Pesquisa 2019. 
O teste de correlação de Pearson mostrou nas escalas de níveis de ansiedade positivo de ambos os gêneros que não há relação significativa entre os níveis de estado de ansiedade, com exceção dos níveis de ansiedade estados da escala negativa de ambos os gêneros.

A partir das abordagens feitas no presente estudo, juntamente com os dados coletados, percebe-se que em eventos competitivos é comum à presença de fatores estressantes que por sua vez podem evocar reações emocionais bem como alterações no comportamento dos participantes. Estas respostas (ansiedade) podem ter magnitudes elevadas (percepção de ameaça), sendo que desta forma pode haver influencias negativas no desempenho do sujeito nos momentos prévios e no momento da realização da atividade, ou seja, da competição.

Os altos níveis de ansiedade estado estão associados a importantes prejuízos funcionais, apesar de estudos avançados e de tentativas na compreensão desses "transtornos", o tratamento da ansiedade ainda constitui um desafio para a prática clínica, embora a relevância do tema seja indiscutível, há poucos estudos que investigam de forma sistematizada o assunto (CARVALHO; FARAH; GALDEANO, 2017).

A ansiedade pode aparecer na pré ou pós-apresentação de dança em um dançarino. Porém, de acordo com
Fabian (2017), o nível de ansiedade que precede uma competição, e aqui no caso, uma apresentação de dança, é um dos fatores de maior influência na qualidade de desempenho do indivíduo.

A fenomenologia da ansiedade é variável, podendo alternar ao longo do tempo, tendo em consideração os episódios impulsionadores (BAPTISTA et.al., 2018). Os principais sintomas podem incluir, por um lado, são a tristeza, a vergonha, a culpa e, por outro, a cólera, a curiosidade, o interesse ou a excitação (BAPTISTA et. al., 2018)

Nesta última tabela 5 verificamos a classificação da amostra conforme os sentimentos relatados pelos bailarinos pré - apresentação de dança. A tabela é bem clara nos seus dados, foram questionadas as categorias de "Absolutamente não", "Um pouco", "Bastante” e "Muitíssimo" dos seguintes sentimentos: calmo, seguro, tenso, arrependido, à vontade, perturbada, estou preocupada, descansado, ansioso, em casa, confiante, nervosa, agitada, pilha de nervos, descontraído, satisfeita, sinto-me preocupada, agitado e confuso, alegre e bem.

Sendo assim faz-se necessário descrever a porcentagem para cada uma das categorias apresentadas. Pode-se destacar na categoria de "Absolutamente não",

Tabela 3. Classificação da amostra conforme os sentimentos relatados pelos bailarinos pré - apresentação de dança.

\begin{tabular}{|c|c|c|c|c|c|c|c|c|}
\hline \multirow[t]{2}{*}{ Sentimentos } & \multicolumn{2}{|c|}{ Absolutam. Não } & \multicolumn{2}{|c|}{ Um Pouco } & \multicolumn{2}{|c|}{ Bastante } & \multicolumn{2}{|c|}{ Muitíssimo } \\
\hline & *Freq. & $* * \%$ & *Freq. & $* * \%$ & *Freq. & $* * 0$ & *Freq. & $* * 0$ \\
\hline Sinto - me calmo & 10 & 38,5 & 09 & 34,6 & 05 & 19,2 & 02 & 7,7 \\
\hline Sinto - me seguro & 06 & 23,1 & 14 & 53,8 & 06 & 23,1 & 00 & 0,0 \\
\hline Sinto - me tenso & 01 & 3,8 & 11 & 42,3 & 12 & 46,2 & 02 & 7,7 \\
\hline Sinto - me arrependido & 16 & 61,5 & 06 & 23,1 & 02 & 7,7 & 02 & 7,7 \\
\hline Sinto - me à vontade & 07 & 26,9 & 08 & 30,8 & 08 & 30,8 & 03 & 11,5 \\
\hline Sinto - me perturbado & 10 & 39,0 & 09 & 35,0 & 06 & 23,0 & 01 & 3,8 \\
\hline Estou preocupado & 06 & 23,1 & 07 & 26,9 & 08 & 30,8 & 05 & 19,2 \\
\hline Sinto - me descansado & 13 & 50,0 & 03 & 11,5 & 10 & 38,5 & 00 & 0,0 \\
\hline Sinto - me ansioso & 02 & 7,7 & 04 & 15,4 & 08 & 30,8 & 12 & 46,1 \\
\hline Sinto - me em Casa & 06 & 23,1 & 08 & 30,8 & 05 & 19,2 & 07 & 26,9 \\
\hline Sinto - me confiante & 02 & 7,7 & 09 & 34,6 & 12 & 46,2 & 03 & 11,5 \\
\hline Sinto - me nervosa & 01 & 3,8 & 06 & 23,2 & 14 & 53,8 & 05 & 19,2 \\
\hline Sinto - me agitado & 02 & 7,7 & 08 & 30,8 & 11 & 42,3 & 05 & 19,2 \\
\hline Estou uma pilha de Nervos & 06 & 23,1 & 05 & 19,2 & 11 & 42,3 & 04 & 15,4 \\
\hline Sinto - me descontraído & 10 & 38,5 & 06 & 23,1 & 04 & 15,3 & 06 & 23,1 \\
\hline Sinto - me satisfeito & 00 & 0,0 & 12 & 46,2 & 09 & 34,6 & 05 & 19,2 \\
\hline Sinto-me preocupado & 06 & 23,1 & 10 & 38,5 & 09 & 34,6 & 01 & 3,8 \\
\hline Sinto - me agitado e confuso & 12 & 46,2 & 06 & 23,1 & 07 & 26,9 & 01 & 3,8 \\
\hline Sinto - me alegre & 02 & 7,7 & 06 & 23,1 & 06 & 23,1 & 12 & 46,1 \\
\hline Sinto - me bem & 02 & 7,7 & 03 & 11,5 & 11 & 42,3 & 10 & 38,5 \\
\hline
\end{tabular}

*Freq. $=$ Frequência $-\%=$ Percentual

Fonte: Pesquisa 2019. 
$(38,5 \%)$ seguido de sinto-me seguro, preocupado, em casa, uma pilha de nervos e estou preocupado que apresentaram $(23,1 \%)$, como também verificou-se no sentimento de sinto-me arrependido (61,5\%), em relação a sinto-me perturbado $(39,0 \%)$ dos bailarinos foram diagnosticado, para sinto-me descansado o percentual obtido foi de $(50,0 \%)$ e para sinto-me agitado e confuso $(46,2 \%)$

A categoria de "Um pouco" que encontra-se com algum desses sentimentos acima citados, o maior índice foi nos seguintes sentimentos: Seguro $(53,8 \%)$, Satisfeito (46.2\%), Tenso (42.3\%). Já na categoria de "Bastante" a porcentagem maior foi Nervosa (53.8\%), Tenso (46.2\%), Confiante (46.2\%). Percebe-se que nessa categoria a porcentagem de $46.2 \%$ prevalece em dois sentimentos contraditórios que seria confiante e tenso.

Ainda temos a categoria de muitíssimo e os que se destacam foram Ansiosa (46.1\%), Alegre (46.1\%), Bem (38.5\%), Nessa categoria de Muitíssimo também possui dois sentimentos que a porcentagem se repete que seria: ansioso e alegre $(46,1 \%)$ sentimentos diferentes como acima destacados.

Os resultados demonstram que a maioria dos bailarinos apresentaram médio nível de estado de ansiedade, os mesmo demonstraram preocupação e ansiedade antes da apresentação de dança, talvez por se sentirem pouco confiantes das suas técnicas e também por causa da tensão que envolve uma apresentação artística, porém foram capazes de lidar com os sentimentos e criar mecanismos de enfrentamento apropriados de forma a amenizar os efeitos negativos da ansiedade e da tensão vivenciados durante a apresentação.

A ansiedade é motivadora e parece ser um aspecto inevitável da condição humana, tratando-se de uma emoção negativa e perturbadora (STROGNAM,1998; CASTRO, 1992). Caracteriza-se tanto pela sensação pessoal e subjetiva de ameaça, como por uma inquietação ou apreensão (GOUVEIA, 2017; VIEIRA, 2012).

0 estado de ansiedade poderia ser observado como um corte transversal temporal na corrente emocional da vida, passando o sujeito a manifestar sentimentos subjetivos de tensão, apreensão, nervosismo e preocupação com ativação do sistema autónomo (SPIELBERG, 1970).
Considerando que a ansiedade pode ser um traço da personalidade, segundo Soares e Martins (2018) dependendo de sua intensidade e duração poderá ser considerada também um transtorno mental, por este fator que escolhemos o inventário de ansiedade estado como referência para esta pesquisa.

Diante destes achados nota-se que os resultados obtidos com esse estudo levam à reflexão sobre a importância de se preparar física e psicologicamente para uma boa apresentação de dança no sentido de criar estratégias eficazes de aprendizagem e de auxiliar os bailarinos a enfrentar situações reconhecidas como de tensão e conflitos.

Nota-se também que este estudo possui importante relevância prática, uma vez que investiga e discute um sentimento comum no processo de atuação dos bailarinos, ou seja, que é notório que a ansiedade estado esta presente em todo situação e que é comum antes de uma apresentação o bailarino sentir tais sentimentos.

Neste aspecto todos os bailarinos que trabalham profissionalmente são responsáveis por conhecer e mensurar fatores que possam interferir nesse processo de aquisição de identidade artística.

\section{CONCLUSÃO}

De acordo com os objetivos traçados conclui-se que acerca dos níveis de ansiedade estado dos bailarinos, houve realmente uma alteração nos estados emocionais desses participantes antes da apresentação. Foi possível verificar alguns sentimentos antes da apresentação, tais como: calmo, seguro, tenso, arrependido, à vontade, perturbada, preocupada, descansado, ansioso, agitado e confuso com escala significativa na pesquisa.

Conclui-se também que os bailarinos antes da apresentação de dança indicaram a presença de níveis de ansiedade, ou seja, apesar da dança não ser um esporte de alto rendimento ela pode desencadear sintomas de ansiedade nas apresentaçõs, ficando evidente que na pré-apresentação os bailarinos passam por alterações nós níveis de ansiedade e que a realização de pesquisas em torno dessa temática pode contribuir para uma ação reflexiva, podendo criar novos métodos e estratégias para diminuir os níveis de ansiedade no momento da apresentação dos espetáculos de dança. 


\section{REFERÊNCIAS}

BABTISTA, T. As Estações do Corpo. São Paulo: Martins Fontes, 2018.

BACKES, M.T.S. et al. Conceitos de saúde e doença ao longo da História sob o olhar epidemiológico e antropológico. Revista de enfermagem. UERJ, Rio de Janeiro, 2017 jan/mar; 17(1): 111-7.

BARLOW, L. G. e Cruz, J. F. A. (2015). Estudo do estresse, da ansiedade e das estratégias de confronto psicológico no handebol de alta competição. Psicologia: teoria, investigação e prática, 2, 523-548.

CARVALHO, R.; FARAH, O.G.D.; GALDEANO, L.E. Níveis de ansiedade de alunos de graduação frente à primeira instrumentação cirúrgica. Revista Latino Americana de Enfermagem, 2017 nov-dez; 12(6):918-23.

CASTRO, E. D. (1992). A dança, o trabalho corporal e a apropriação de si mesmo. Revista de Terapia Ocupacional, 3(1/2), 24-32.

FABIAN, J. (2017). Psicología de la Actividad Física y del Deporte. Espanha: McGraw- Hill.

GOUVEIA, F. S. Programa de Promoção de Competências Psicológicas para a optimização do desempenho e promoção do bem-estar em alunos de dança. 2017.60f. Dissertação (Mestrado Integrado em Psicologia) - Faculdade de Psicologia e de Ciências da Educação, Universidade de Lisboa.

JUSSARA, J. T.(2017). Dança na Escola: Benefícios e contribuições na fase pré-escolar. 0 Portal dos Psicólogos. Recuperado a partirhttp://www.psicologia.pt/artigos/textos/TL0046.pdf

MAGILL, R., A. Aprendizagem Motora: conceitos e aplicações. São Paulo: Edgard Blücher. 1984.
MARCONI, M. D; LAKATOS, E. M. Fundamento de Metodologia Cientifica. São Paulo: Atlas S.A, 2015.

MELO, A. Ansiedade e depressão nos melhores alunos. 2004. [Citado em 2006 nov. 25]. Disponível em: http://www. netprof.pt/netprof/servlet/getDocumento? id_versao=12506.

PRIMO, A. C. S., PRADO, W. L., PRADO, M. C. L. Ansiedade em bailarinos profissionais nas apresentações de dança. Revista da Faculdade de Educação Física da UNICAMP, Campinas, v. 8, n. 3, p. 146-155, set/dez. 2018.

SOUSA, F. N. G.; MARIANI, M. E., SAMULSKI, D. M. Análise do nível de estresse e da ansiedade em bailarinos e bailarinas profissionais na pré-estreia de um espetáculo de dança. 68f. 2016. Dissertação (Mestrado) - Universidade Católica de Brasília, Brasília, 2016.

SPIELBERGER, C.D.; GORSUCH, R.L.; LUSHENE, R.E. Manual for the state-trait anxiety inventory. Palo Alto: Consulting Psychologist Press; 1970.

STRONEMAN, K (1998). A psicologia da emoção (4ํeㄹição), Lisboa: climepese.

SOARES, A.B.; MARTINS, J.S.R. Ansiedade dos estudantes da expectativa do exame vestibular, Paideia, jan-abr, 2018, v. 20, n. 45 , p. $57-62$.

THOMAS, J. R.; NELSON, J. R. Métodos de Pesquisa em Ativi-

VIEIRA, M., T. Psicologia do Esporte: a ansiedade e o estresse pré-competitivo. Portal dos Psicólogos, 2012. Apresenta informações relacionadas a psicologia. Disponível em: $<W W W$. psicologia.com.pt> Acesso em: 10 set. 2019. 